\title{
A metabolic profiling analysis of degenerative intervertebral disc disease in a rabbit model via GC/TOF-MS
}

\section{Xiaolin Wu}

medical department of qingdao university

Hongfei Xiang

Qingdao Women and Childrens Hospital

\section{Guoqing Zhang}

the affiliated hospital of qingdao university

Yougu $\mathrm{Hu}$

the affiliated hospital of qingdao university

yan wang

Qingdao Women and Childrens Hospital

Bohua Chen ( $\square$ bhchen@hotmail.com )

Affiliated Hospital of Qingdao University https://orcid.org/0000-0002-5400-4352

\section{Research article}

Keywords: Intervertebral disc degeneration;Metabolomics;GC/TOF-MS(gas chromatography/time-of-flight mass spectrometry);Total ion chromatograms;PLS-DA(partial least squares-discriminant analysis)

Posted Date: November 14th, 2019

DOI: https://doi.org/10.21203/rs.2.17337/v1

License: (c) (1) This work is licensed under a Creative Commons Attribution 4.0 International License.

Read Full License 


\section{Abstract}

Background: To establish an analytical method for studying intervertebral disc metabolomics based on gas chromatography/time-of-flight mass spectrometry (GC/TOF-MS). Methods: To establish a rabbit model of intervertebral disc degeneration, the intervertebral discs of six New Zealand white rabbits were punctured. GC/TOF-MS was applied to analyze degenerated discs of the six model group animals and six similar New Zealand white rabbits considered as the control group. Pattern recognition and nonparametric test analyses were utilized to evaluate the data as well as screen for and identify significant biomarkers. Metabolites and metabolic pathways relevant to associated pathological processes were studied. Results: We established a rabbit model of intervertebral disc degeneration and an orthogonal partial least squares-discriminant analysis (OPLS-DA) disease-distinguishing model of intervertebral disc degeneration for contrast against the typical physiological state of intervertebral discs. Expression of six metabolites in degenerated and normal intervertebral discs of New Zealand white rabbits was evaluated. Levels of four metabolites expressed in the model group were significantly higher than in the control group, while two other metabolites were expressed at significantly lower levels in the model group as compared to the control group. Conclusions: This paper demonstrates that metabolic profiling of both degenerated and normal intervertebral rabbit discs is a feasible method of metabolomic analysis. Via in-depth filtering of characteristic metabolites, we found a high correlation between metabolic variations and intervertebral disc degeneration. Further studies on endogenous metabolites and pathways involved in intervertebral disc degeneration will provide a better understanding of associated molecular mechanisms and lay foundations for effective clinical treatment.

\section{Background}

Intervertebral disc degeneration (IDD) is the primary etiology and pathological basis for spinal disc herniation and other degenerative diseases of the spine. Although this condition seriously affects patient quality of life, has a high incidence and morbidity, and is the commonest cause of lower back pain seen in clinical settings, the precise pathological mechanisms of this condition have yet to be elucidated $[1,2$, 3]. . The intervertebral disc primarily consists of extracellular matrix (ECM) and is rich in water, collagen, proteoglycans and other such materials. Recently, it has been recognized that IDD is caused by a serious, chronic metabolic imbalance in the ECM itself. Abnormal metabolism within disc tissue results in deficiencies of proteoglycans and type $\nabla$ collagen. Although research and discussion of IDD in histological, molecular biological, immunological, and physical contexts has been plentiful, reports from a metabolomic perspective, to date, are scarce.

Metabolomics is rapidly emerging as a branch of systems biology incorporating genomics, transcriptomics and proteomics. This field of study focuses on investigating metabolite profiles and dynamics when biological systems are stimulated or interfered with [5]. Compared with other branches of systems biology, metabolomics presents unique advantages. Since changes in gene expression affect expression of associated protein and inevitably influence metabolic processes, metabolomics is therefore the most appropriate analytical method to study phenotypes and metabolic responses to phenotypic 
changes. In addition, as biological metabolites possess versatile characteristics, identifying and perfecting modern analytical methods for evaluation of one or a class of metabolites is preferable to relying on older analytical modalities [6]. Finally, since it is much more difficult to isolate and study metabolites than genes and proteins, metabolomics offers a novel methodology of biological phenotype evaluation and represents a great advance in systems biology.

Nuclear magnetic resonance (NMR), chromatography combined with mass spectrometry and spectrum technology are the main evaluative methods utilized in metabolomic analysis. Gas chromatography combined with mass spectrometry was initially applied in metabolomic hyphenated techniques. In addition to possessing highly sensitive and precise detection capabilities, a standard metabolite spectral library is readily available for data reference and comparison.

Gas chromatography / time-of-flight mass spectrometry (GC / TOF-MS) technology is mainly utilized for separation and identification of volatile substances. GC / TOF-MS can detect and analyze hundreds of metabolites without necessitating application of the pre-edit mass spectrometry acquisition method. In addition, the dynamic range of compounds tested for is much wider than in other metabolomic analysis techniques. With the rapid development of chemical derivatization in recent years, interactions of many volatile gaseous substances with animal metabolic processes have been studied using GC / TOF-MS [78].

In this study, both degenerated and normal rabbit intervertebral discs were evaluated using GC/TOF-MS. We analyzed metabolic profile dynamics in the setting of intervertebral disc degeneration and explored differences in metabolite expression between experimental and control groups. This study also investigated molecular mechanisms involved in the pathogenesis of IDD and potential clinical values of relevant metabolites.

\section{Methods}

A total of 12 healthy, New Zealand white rabbits (age, three months; weight, two-three kg) were obtained from Kangda Biological Technology Co. SCXK (Lu) 20120005 (Qingdao, China) and housed in an animal care facility. All animals experiment has been approved by the Medical (Ethics) Committee of the Affiliated Hospital of Qingdao University (QDFY WZ 2018-9-14-02)Spinal MRI excluded pre-existing deformities in all subject animals. The New Zealand white rabbits were randomly divided into a control group $(n=6)$ and a model group $(n=6)$. Animals were treated in compliance with accepted ethical standards [Ethical issues in animal experimentation, 2009].

\section{Puncture surgery}

Each model group rabbit was anesthetized with $3 \%$ sodium pentobarbital ( $30 \mathrm{mg} / \mathrm{kg})$ via ear vein injection. The rabbit was fixed to a CT scanner bed in a left lateral position while under general anesthesia, maintaining a straightened lumbar spine. Intervertebral disc (L1-7) CT scan was subsequently performed, with Kirschner wire positioning in the $L 4 / 5$ intervertebral space. The $L 4 / 5$ position was 
marked on the rabbit's skin with a pen and CT-guided intervertebral disc puncturing was then performed. When CT confirmed that the 18-gauge needle point was located within the nucleus pulposus at a depth of $5 \mathrm{~mm}$ (Figure 1.1), the needle was withdrawn. The target disc was punctured thrice in the aforementioned manner. After the final puncture, a small amount of residual nucleus pulposus was visually noted within the puncture needle accompanied by a milky-white gelatinous material. After completion of the procedure, puncture wounds were disinfected with iodine and covered with bandaging materials. An intramuscular injection of penicillin $(200,000 \mathrm{U})$ was administered to all animals, and the rabbits were permitted to move freely about their cages. No animal suffered death or infection due to experimental procedures.

\section{Specimen collection}

Four weeks after surgery, rabbits from both groups were anesthetized with $3 \%$ sodium pentobarbital (30 $\mathrm{mg} / \mathrm{kg}$ ) via ear vein injection. After induction of general anesthesia, MRI was performed on all rabbits. Imaging included a lumbar sagittal scan; fast spin echo (FSE), T2-weighted imaging sequences, and scanning lines passing through the center of imaged intervertebral discs.

Specific MRI parameters included: repetition time, TR 3000 ms; echo time, TE 125ms; horizon, $180 \mathrm{~mm}$; thickness, $3 \mathrm{~mm}$; layer spacing, $1 \mathrm{~mm}$; horizon, $16 \mathrm{~cm} \times 16 \mathrm{~cm}$; matrix, $256 \times 196$; and an excitation number of 2. Imaging was performed over a period of five hours and 36 minutes. Imaging data acquired was transmitted to a picture archiving and communication system (PACS). T2W1 signal intensity of each rabbit's $L 4 / 5$ intervertebral disc was acquired while referencing the Pfirrmann grading system (Figure 1.2) [10]. Data concerning grading and assessment of the severity of disc degeneration in each rabbit is shown in Table 1.1. All MRI data was compiled by physicians with extensive diagnostic radiologic experience.

\section{Methods of Euthanasia and subsequent processing}

The sodium pentobarbital was formulated into a $3 \%$ physiological saline solution, fully dissolved in a $65^{\circ} \mathrm{C}$ water bath, and inhaled into a $20 \mathrm{ml}$ syringe for use. The experiment was fixed on a holder with a rabbit, and the rabbit ear is disinfected with a $1 \%$ polyvinylpyrrolidone solution. The indwelling needle was inserted into the ear vein of the rabbit, the medical film was fixed, and the rabbit head fixing frame was released to make it in a good breathing environment, at intervals of $10 \mathrm{~min}$, according to the conventional anesthetic dose of $50-70 \mathrm{mg} / \mathrm{Kg}$, the ear vein was administered intravenously, Check that the animal was in deep anesthesia. Continue to dose to 3 times the anesthetic dose, Check the rabbit for no spontaneous breathing for 2-3min, no heartbeat and blink reflex,Convinced that the execution was successful.

Rabbits were sacrificed 24 hours following MRI. L4/5 intervertebral discs of each experimental animal were isolated (Figure 1.3) and disc samples weighing approximately $40 \mathrm{mg}$ each were sectioned. $1 \mathrm{~mL}$ of methanol: water (9:1) solution was added to the samples followed up with $15 \mu \mathrm{L}$ of ribose alcohol solution $(0.2 \mathrm{mg} / \mathrm{mL})$. The mixture was sufficiently homogenized, products ultrasonically extracted 
thrice with each extraction lasting 15 minutes, and finally centrifuged at $13000 \mathrm{r} / \mathrm{min}$ for 20 minutes. The supernatant was then refrigerated at $-80^{\circ} \mathrm{C}$ for further analysis.

\section{Sample preprocessing}

Thawed supernatant was blow dried with $\mathrm{N} 2$ gas at $45^{\circ} \mathrm{C}$ and $40 \mu \mathrm{L}$ of methoxyamine hydrochloric acid solution $(20 \mathrm{mg} / \mathrm{mL})$ was added to the extract. The solution was mixed thoroughly, maintained for 90

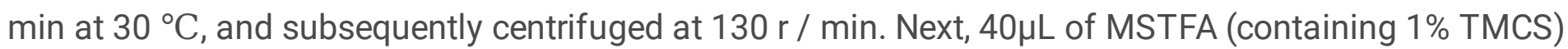
was added. The solution was mixed thoroughly and maintained in an air bath at $37^{\circ} \mathrm{C}$ for 30 minutes and then kept at room temperature for 120 minutes. Finally, the mixture was refrigerated at $4^{\circ} \mathrm{C}$ for later measurement.

\section{GC / TOF-MS analysis conditions}

Instrument model: GCT mass spectrometer (Waters, USA); capillary column type: DB-5; injection volume: $1 \mu \mathrm{L}$; Inlet temperature: $250^{\circ} \mathrm{C}$; shunt mode: no shunt; carrier gas: helium; airflow patterns: constant current; inlet mode: back injector; detector type: MCP; column flow rate: $1.0 \mathrm{~mL} / \mathrm{min}$; initial column temperature: $70^{\circ} \mathrm{C}$; maximum column temperature: $280^{\circ} \mathrm{C}$; programmable temperature: $70^{\circ} \mathrm{C}(1 \mathrm{~min})$; $5^{\circ} \mathrm{C} / \mathrm{min}$ to $280^{\circ} \mathrm{C}(10 \mathrm{~min})$; solvent delay: $330 \mathrm{~s}$; scan range: $50 \sim 800 \mathrm{Da}$; scan rate: $3.1 \mathrm{r} / \mathrm{min}$; ion source temperature: $220^{\circ} \mathrm{C}$; ionization voltage: $70 \mathrm{eV}$; connecting rod temperature: $250^{\circ} \mathrm{C}$.

\section{UHPLC-QTOF-MS analysis}

UHPLC-QTOF-MS analysis was performed with an Agilent 6540 ultra-high definition accurate mass quadruple time-of-flight spectrometer with UHPLC capabilities (UHPLC-QTOF-MS, Agilent Technologies, U.S.A.). A UPLC C18 analytical column (2.1 mm × $100 \mathrm{~mm}$, I.D. $1.7 \mu \mathrm{m}$, ACQUITY UPLC®BEH, Waters, U.S.A.) was used for separation, coupled with a C18 pre-column $(2.1 \mathrm{~mm} \times 5 \mathrm{~mm}$, I.D. $1.7 \mu \mathrm{m}$, VanGuardTM BEH, Waters, U.S.A.) at $20^{\circ} \mathrm{C}$. The mobile phase was characterized by a mixture of water (A) and acetonitrile (B), both containing $0.1 \%$ formic acid, with an optimized linear gradient elution as follows: $0-5 \mathrm{~min}, 10-35 \% \mathrm{~B} ; 5-25 \mathrm{~min}, 35-55 \%$ B; $25-28 \mathrm{~min}, 55-85 \% \mathrm{~B} ; 28-30 \mathrm{~min}, 85-100 \% \mathrm{~B}$. The injection volume was $4 \mu \mathrm{L}$, and the flow rate was set at $0.35 \mathrm{~mL} / \mathrm{min}$. Mass spectra were acquired in negative mode by scanning from 100 to 1700 in a mass to charge ratio $(\mathrm{m} / \mathrm{z})$. MS analysis was performed under the following operative parameters: dry gas temperature $300{ }^{\circ} \mathrm{C}$, dry gas (N2) flow rate 5 $\mathrm{L} / \mathrm{min}$, nebulizer pressure $30 \mathrm{psi}$, Vcap 3000 , nozzle voltage $500 \mathrm{~V}$, and fragmentor voltage $200 \mathrm{~V}$.

\section{Data preprocessing}

Chroma TOF software (Version 4.5, LECO Corporation, USA) was used for automatic peak detection, internal standard setting and calculation of the special compound peak area. In total, 315 peaks were detected. Compounds were identified by comparing mass spectra with the mass spectral library of the National Institute of Standards and Technology (National Institute of Standard and Technology, NIST) and related verification standards. 
Statistical analysis

Based on spectral similarities and retention time-shift corrections, the base peak ion integrated signal was generated using MarkerLynx software. Multivariate pattern recognition analysis of obtained data was performed using SIMCA-P10.0 software (Umetrics, Umea); PCA and PLS-DA were applied to the $\mathrm{GC} / \mathrm{TOF}$ MS spectral data set. The t-test was applied for comparison of the two groups; $\mathrm{P}<0.05$ was considered to indicate a statistically significant difference.

\section{Results}

Total ion chromatograms, TIC

The characteristic GC / TOF-MS TIC data obtained for both experimental groups are shown in Figure 2.1; a significant difference in levels and sizes is easily apparent between the chromatographic peaks of the two groups.

\section{$P C A$ and PLS-DA}

Dimension-pattern recognition is necessary for noise elimination from large amounts of complex biological data obtained and analyzed for potential biological significance [11]. PCA score plots of GC/TOF MS data (Figure 2.2.) reveal a separation between two groups to be unclear (R2X=0.631, Q2= $0.1438,2$ principal components). To enhance the recognition intensity of classification variables and underscore a clear separation between the experimental and control groups, PLS-DA methods were applied. Results are shown in Figure 2.3. PLS-DA score plots of GC/TOF MS data reveal an obvious separation between the two groups, with the model possessing a good degree of separation and predictability ( $R 2 X=0.528, \mathrm{R} 2 \mathrm{Y}=0.807, \mathrm{Q} 2=0.494 ; 2$ principal components). There was a significant difference in metabolic measurements between the two groups.

\section{UHPLC-TOF MS Non-target Screening.}

Accurate UHPLC-TOF MS data were also processed in a non-target manner utilizing the ChromaLynx Application Manager. The availability of a QTOF instrument made it feasible to perform MS/MS experiments for both samples and even to further confirm the identity of detected compounds. Results were similar to those obtained from GC-TOF MS. As shown as in Figure 3, a significant difference in levels and readings is easily apparent between the chromatographic peaks of the two groups. Deviations in measured masses of all product ions were lower than $2.1 \mathrm{mDa}$. Additionally, when relative abundances of 6 different metabolites in suspected positive samples were compared with reference values, all deviations were within proposed limits. Finally, retention times for reference standards and sample peaks were also compared, yielding a deviation of $<2.3 \%$. Samples were thereby confirmed via QTOF with high reliability to be positive for intervertebral disc content.

\section{Metabolites}


To identify metabolites responsible for noted differences, PLS-DA loading (Figure 2.4) and variable importance in projection (VIP) plots (Table 2.1) were generated. A total of 42 contributing features were obtained from the PLS-DA both plots. Inspection of the VIP plot with respect to separation revealed six features to possess VIP values of $>0.8$ ( $P \llbracket 0.05$ ) (Table 2.2). Compared with the control group冈levels of lactic acid, alayl-alanine, glutamic acid, and oxoproline were obviously elevated. The content of pinitol glucose-1-phosphate, however, was noted to be decreased (Table2.1-2.2).

\section{Discussion}

IDD is an important etiology of neck pain, back pain, and similar complaints commonly seen in the clinical setting [12]. Approximately $80 \%$ of patients complain of suffering symptoms of lower back pain at least once in their lifetime [13]. IDD has become recognized as a chronic process playing a major role in the pathogenesis of degenerative spinal disease. This condition not only alters the type, number and morphology of disc cells, but also influences changes in disc tissue on a molecular biological level. Integrated research from a metabolomic perspective is obviously indispensable for efficiently analyzing complex metabolic interactions within disc tissue in the setting of IDD, as well as studying various internal and external influencing factors on disc physiology.

Metabolomics, a rapidly developing branch of systems biology based on group index analysis involving high-throughput, high-sensitivity detection of sample and data processing with a goal of information modeling and system integration. Metabolomics involves the detection and analysis of metabolite profiles and their dynamic changes when biological systems are affected by various stimulating or interfering factors. Metabolomics involves a comprehensive analysis of small molecule metabolites $(<1$ $\mathrm{kDa}$ ) within an organism, tissue, or a specific biological sample [14-15].

In contrast to other analytical methodologies, metabolomics has clear specific advantages [16]. First, minute changes in gene and protein expression are often difficult to detect, but such small changes can produce great increases of involved metabolites readily detected by biochemical techniques. Secondly, metabolomic evaluation does not necessitate complex whole genome sequencing and does not require cumbersome establishment of proteomic expression sequence databases. Thirdly, the number of metabolites in the human body is relatively small in comparison to the number of genes or proteins considered for study, and metabolomics offers an efficient and focused approach for analysis of these metabolites. In addition, detected metabolites are easily correlated to phenotypes of living organisms and provide information on biochemical processes by revealing accurate "bigger pictures" of substrates and product interactions during cellular metabolism. Fields such as drug development, disease research, botanical biochemistry, and microbiology are being approached from a new perspective thanks to these emerging analytical methods [17].

MS, with its high sensitivity and specificity, can rapidly analyze and measure a variety of different metabolites. However, not all metabolite categories produce an acceptable spectrometric resolution and are therefore distinguished according to respective degrees of compound ionization. In addition, selection 
of suitable separation techniques may compensate for some minor shortcomings of poor MS resolution [23]. In order to identify and isolate the compounds in a comprehensive and independent manner, researchers have improved MS by combining it with GC. GC-MS technology has been widely applied and rapidly developed in metabolomic research due to its numerous advantages [24]. GC/TOF-MS can detect and analyze hundreds of metabolites at a time without necessitating a pre-edit collection method otherwise common when relying solely on MS. This combined test offers a much higher range of subject compounds than other metabolomic analytical techniques do. Traditional GC / TOF-MS are currently used in El (ion source ionization) mode. Characteristics of this testing modality include a stable performance and efficient collection of a large amount of fragmented information regarding subject compounds.

Identification of novel compounds generally involves analysis of both target and unknown substances [25-26]. Identification of unknown compounds usually requires application of auxiliary software and meticulous cross-referencing of related databases. Throughout the process of data analysis, data obtained in an experiment can be analyzed continuously for extremely efficient compilation of information without requiring implementation of re-collection methods.

\section{Conclusions}

In this experiment, normal and degenerated rabbit intervertebral discs were analyzed in depth. GC/TOFMS was applied to study discs of both model and control groups; meanwhile, the established UHPLCQTOF-MS method used in this study as well as potential differentiating markers were utilized to profile and evaluate both model and control groups. Each analytical technique yielded similar results. Six characteristic metabolites were selected from both degenerated and normal discs (lactic acid, alanylalanine, glutamic acid, oxoproline, pinitol, and glucose-1-phosphate). According to the Kegg path database (http://www. Genome. Jp/kegg/), these six substances are mainly involved in the metabolism of glucose and amino acids.

Due to chronic degeneration and calcification of upper and lower vertebral endplates, intervertebral disc permeability and areas of effective penetration decrease significantly [27]. In addition, disc degeneration results in impairment of substance exchange with the surrounding physiological environment and reduction of intradiscal oxygen content. These changes can strengthen anaerobic metabolism within the cells of the nucleus pulposus and result in a great amount of lactic acid (and other metabolic byproduct) accumulation, leading to the eventual formation of a highly acidic microenvironment. [28-29]. Of note, it has been reported that lactic acid exerts adverse effects on disc ECM, negatively influences cell viability, and is considered a metabolic biomarker of discogenic lower back pain [30, 31, 32].

This study also found levels of glutamate, propionyl-alanine, and proline oxygen to be significantly elevated in the model group as compared with the control group. These findings may be due to increasing hydrolytic activity within the main components of disc ECM (collagen and proteoglycan). Our data consider matrix metalloproteinase (MMP) hydrolysis of collagen and other pathological intervertebral 
disc matrix components under a variety of diseased conditions [33]. Proteoglycan degradation is closely related to disc degeneration, degradation product accumulation, and neurotransmitter (i.e. glutamate) release and buildup within the local microenvironment, eventually affecting nerve function [34-35]. Harrington found that high perineural concentrations of glutamate can activate glutamate receptors associated with neural activity, and considered it a primary reason of lumbar disc degeneration resulting in lower back pain. Increases in glutamic acid concentration within degenerated intervertebral discs therefore have great potential to be used as biomarkers of IDD.

Glucose-1-phosphate is an important component within the citric acid cycle and a major source of energy. This study found levels of glucose-1-phosphate in degenerated intervertebral discs to be lower than in normal discs. This may have been due to increased aerobic metabolism closely related to inflammatory reactions involved in disk degeneration [37]. These findings are also consistent with a chronic absence of glucose. This intitially leads to necrosis of the nucleus pulposus and eventually results in disc degeneration [38].

Moreover, we found expression of metabolites within intervertebral discs to be significantly different between experimental and control groups. However, no evidence supporting an association between methyl-inositol and IDD was elucidated and further research on any potential relationship is required.

Successful analysis of metabolites within a metabolic profiling model suggests metabolomic investigation to be a feasible approach for studying degenerative disc disease and provides a novel perspective from which to further study involved pathophysiology. Furthermore, metabolomic investigation is bound to provide a new basis for the understanding of the pathological basis of intervertebral disc degeneration from the genetic and protein levels.

\section{Abbreviations}

1.IDD: Intervertebral disc degeneration

2.OPLS/DA:Orthogonal partial least squares-discriminant analysis

3.NMR: Nuclear magnetic resonance

4.PACS:Picture archiving and communication system

5.GC/TOF-MS: Gas chromatography/time-of-flight mass spectrometry

6.TIC:Total ion chromatograms

7.MSTFA:N-Methyl-N-(trimethylsilyl)trifluoroacetamide

8.UHPLC-TOF MS:Combining Ultrahigh performance liquid chromatography-ion mobility spectrometrymass spectrometry and time-of-flight mass spectrometry 
9.PLS-DA: Partial least squares-discriminant analysis

10.ECM:Extracellular matrix

11.MMP:Matrix etallopromteinase

\section{Declarations}

- Ethics approval and consent to participate

This study has been approved by the Medical (Ethics) Committee of the Affiliated Hospital of Qingdao University (QDFY WZ 2018-9-14-02), and all participating animals are in compliance with ethical standards

- Consent for publication

Not applicable

- Availability of data and material

The datasets used and/or analysed during the current study are available from the corresponding author on reasonable request.

- Competing Interests:

The authors declare that they have no competing interests

- Funding

This work was supported by the National Natural Science Foundation of China[81802190®81772412] (Provide experimental consumable support); Shandong Medical and Health Technology Development Plan Project[2017WS033](Provide instrument and data analysis support) \and Youth Fund Project of the Affiliated Hospital of Qingdao University [2017](Provide administrative license and project approval). All funders did not participate in the content of this experiment, they had given different levels of support in the project funding and equipment, we stated that there was no conflict of interest and copyright transfer issue with the funder.

\section{- Authors' contributions}

XLW:Experimental design execution and writing of the full paper,HFX:Organize experiments and get the corresponding data,GQZ:Animal experiment and statistical analysis,YGH:Literature search and writing comments;YW:Trial project approval and article language part modification;BHC:The overall project leader and experimental opinion presenter

all authors have read and approved the manuscript. 
- Acknowledgement

The authors thank Professor Yong Liu for his helpful advice regarding researching unpublished literature.

\section{References}

[1]Stewart WF, Ricci JA, Chee E. Lost productive time and cost due to common pain conditions in the US workforce . JAMA, 2003, 290(18):2443-2454.

[2]Choi KC, Kim JS, Kang BU. Changes in back pain after percutaneous endoscopic lumbar discectomy and annuloplasty for lumbar disc herniation: a prospective study . Pain Med, 2011, 12(11):1615-1621.

[3]Wu H, Xue R, Lu C. Metabolomic study for diagnostic model of oesophageal cancer using gas chromatography/mass spectrometry. J Chromatogr B Analyt Technol Biomed Life,2009, 877:3111-3117.

[4]Urban MR, Fair JC, Etheington PJ. Electrochemical measurement of transport into scoliotic intervertebral discs in vivo using nitrous oxide tracer. Spine (Phila Pa 1976), 2001区26:984-990.

[5]Nicholson JK, Lindon JC, Holmes E.'Metabonomics': understanding the metabolic responses of living systems to pathophysiological stimuli via multivariate statistical analysis of biological NMR spectroscopic data. Xenobiotica, 1999, 29(11): 1181-1189.

[6]Monton MRN, Soga T. Metabolome analysis by Capillary Electrophoresis-Mass Spectrometry. Journal of Chromatography A, 2007, 1168: 237-246.

[7]Gou X, Tao Q, Feng Q. Urinary metabonomics characterization of liver fibrosis induced by CCl4 in rats and intervention effects of Xia Yu Xue Decoction. J Pharm Biomed Anal, 2013, 74:62-65.

[8]Gou X, Tao Q, Feng Q. Urine metabolic profile changes of CCl4-liver fibrosis in rats and intervention effects of Yi Guan Jian Decoction using metabonomic approach. BMC Complement Altern Med, 2013, 13:123-135.

[9]R J B Peters, A A M Stolker, J G J Mol. Trends Anal. Chem, 2010, 29(11):1250-1268.

[10]Pfirrmnann C W, Metzdorf A, Zanetti M. Magnetic resonance classification of lumbar intervertebral disc degeneration. Spine, 2001, 26(17):1873-1878.

[11]Eriksson L, Antti $\mathrm{H}$, Gottfries J. Using chemometrics for navigating in the large date sets of genomics, proteomics, and metabonomics(gpm). Anal Bioanal Chem, 2004, 380(3):419-429.

[12]Adams M A, Roughley P J. What is intervertebral disc degeneration, and what causes it?. Spine, 2006, 31(18): 2151-2161.

[13]Waddell G Low back pain: a twentieth century health care enigma. Spine, 1996, 21(24): 2820-2825. 
[14]Nicholson JK, Lindon JC, Holmes E. 'Metabonomics': understanding the metabolic responses of living systems to pathophysiological stimuli via multivariate statistical analysis of biological NMR spectroscopic data. Xenobiotica, 1999, 29(11): 1181-1189.

[15]Fiehn O, Kopka J, Dormann P. Metabolite profiling for plant functional genomics. Nat Biotechnol, 2000, 18(11): 1157-1161.

[16]Guowang Xu, Xin Lu, Shengli Yang. Advances in metabonomics. Journal of Chinese Academy of Medical Sciences, 2007, 29(6): 701-711.

[17]Zhehua Zhang, Huinan Ge. Progress of metabonomics in the diagnosis of digestive tract diseases. Journal of Qilu Medical Journal, 2014, 29(3):279-280.

[18]Fiehn O. Extending the breadth of metabolite profiling by gas chromatography coupled to mass spectrometry. Trends in Analytical Chemistry, 2008, 27(3): 261-269.

[19]F Hernandez, T Portoles, E Pitarch . Trends Ana1. Chem. , 2011, 30, (2):388-400.

[20]F Hernandez, T Portoles, E Pitarch . Mass Spectrom , 2009, 44(1):1-11.

[21]Goupille P, Malcom $\otimes$. Jayson, Jean-pierre Valat. Matrix metalloproteinases: the clue to intervertebral disc degeneration. Spine. 1998, 23:1612-1626.

[22]Grunhagen T, Wilde G, Soukane DM. Nutrient supply and intervertebral disc metabolism. J Bone Joint Surg Am. 2006, 88(Suppl 2):30-35.

[23]Dettmer K, Aronov PA, Hammock BD. Mass spectrometry-based metabolomics. Mass Spectrom Rev, 2007, 26(1): 51-78.

[24]Ackermann BL, Hale JE, Duffin KL. The role of mass spectrometry in biomarker discovery and measurement. Curr Drug Metab, 2006, 7(5): 525-539.

[25]Dunn WB, Ellis DI. Metabolomics: current analytical platforms and methodologies. Trac Trends Anal Chem, 2005, 24(4): 285-294.

[26]Hollywood K, Brison DR, Goodacre R. Metabolomics: current technologies and future trends. Proteomics, 2006, 6(17): 4716-4723.

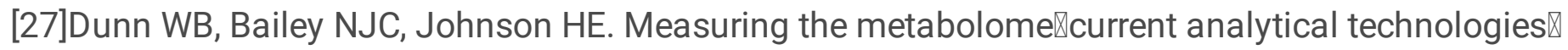
Analyst $₫ 2005 \bigotimes 130(5) \llbracket 606-625$.

[28]Monton MRN, Soga T. Metabolome analysis by Capillary Electrophoresis-Mass Spectrometry. Journal of Chromatography A, 2007, 1168: 237-246. 
[29]Benneker LM, Heini PF, Alini M. 2004 Young Investigator Award Winner: vertebral endplate marrow contact channel occlusions and intervertebral disc degeneration. Spine. 2005, 30(2): 167-173.

[30]S. R. Bibby, J. P. Urban. Effect of nutrient deprivation on the viability of intervertebral disc cell . Eur. Spine J., 2004, 13, 1369-6701.

[31]D. M. Soukane, S. A. Shirazi-Adl, J. P. Urban. Investigation of solute concentrations in a 3D model of intervertebral disc . Eur. Spine J., 2009, 18, 254-262.

[32]K. R. Keshari, J. C. Lotz, T. M. Link, S. Hu, S. Majumdar and J. Kurhanewicz, Lactic acid and proteoglycans as metabolic markers for discogenic back pain, Spine, 2008, 33(3), 312-317.

[33]Bachmeier BE, lancu CM, Jochum M, et al. Matrix metalloproteinases in cancer: comparison of known and novel aspects of their inhibition as a therapeutic approach. Expert Rev Anticancer Ther, 2005, 5(1):149-163.

[34]J. E. Heuttner, Glutamate receptor channels in rat DRG neurons: activation by kainite and quisqualate and blockade of desensitization by Con A, Neuron, 1990, 5, 255-267.

[35]M. A. Adams and P. J. Roughley, What is intervertebral disc degeneration, and what causes it, Spine, 2006, 31(18), 2151-2161.

[36]J. F. Harrington, A. A. Messier, D. Bereiter. Herniated lumbar disc material as a source of free glutamate available to affect pain signals through the dorsal rood ganglion. Spine, 2000, 25(8), 929-936.

[37]M. Gårseth, U. Sonnewald, L. R. White. Metabolic changes in the cerebrospinal fluid of patients with lumbar disc herniation or spinal stenosis. Neurosci. Res., 2002, 69(5), 692-695.

[38]H. A. Horner, J. P. Urban. Volvo Award Winner in Basic Science Studies: effect of nutrient supply on the viability of cells from the nucleus pulposus of the intervertebral disc, Spine, 2001, 26, 2543-2549.

\section{Tables}

Table 1

\begin{tabular}{|c|c|c|c|c|c|}
\hline Group/Grade & Ggrade & $\square$ grade & $\square$ grade & $\square$ grade & Ggrade \\
\hline Control group & 6 & 0 & 0 & 0 & 0 \\
\hline Model group & 0 & 0 & 4 & 2 & 0 \\
\hline
\end{tabular}

\section{Table 1.1 Postoperatively four weeks the MRI Pfirrmann grade of disc degeneration}

\section{Table2.1}




\begin{tabular}{|c|c|c|c|c|}
\hline number & Compounds & $\mathrm{RT}(\mathrm{s})$ & $\mathrm{VIP}$ & $P$ \\
\hline 1 & Unknown Compounds ${ }^{a}$ & 395.492 & 1.0939 & 0.141026 \\
\hline 2 & lactic ac & 418.2 & 1.60741 & 0.005393 \\
\hline 3 & Valine(1TMS) & 453.025 & 1.66364 & 0.237421 \\
\hline 4 & Unknown Compounds ${ }^{a}$ & 469.575 & 2.94226 & 0.209532 \\
\hline 5 & Alanine(2TMS) & 469.727 & 2.1519 & 0.155046 \\
\hline 6 & Isoleucine & 551.983 & 1.42128 & 0.16944 \\
\hline 7 & Valine(2TMS) & 638.892 & 1.35258 & 0.44826 \\
\hline 8 & Urea $^{\mathrm{b}}(2 \mathrm{TMS})$ & 699.25 & 3.85859 & 0.299056 \\
\hline 9 & Serine(2TMS) & 708.9 & 1.59204 & 0.101732 \\
\hline 10 & Phosphoric acid(3TMS) & 717.417 & 2.08962 & 0.125222 \\
\hline 11 & Leucine(2TMS) & 727.483 & 1.75192 & 0.380003 \\
\hline 12 & Phosphoric acid(3TMS) & 733.233 & 3.76547 & 0.009287 \\
\hline 13 & glycerin $^{\mathrm{b}}$ (3TMS) & 736.317 & 5.1556 & 0.059845 \\
\hline 14 & Prolin & 765.892 & 2.15939 & 0.650301 \\
\hline 15 & Glycine(3TMS) & 782.4 & 2.84485 & 0.547797 \\
\hline 16 & alanyl-alanine $\mathrm{b}^{\mathrm{b}}$ & 864.525 & 2.71308 & 0.01851 \\
\hline 17 & Serine(3TMS) & 865.87 & 1.02363 & 0.656996 \\
\hline 18 & Threonine(3TMS) & 904.35 & 1.31295 & 0.501199 \\
\hline 19 & Unknown Compounds ${ }^{a}$ & 1082.45 & 1.63029 & 0.146786 \\
\hline 20 & oxoproline(2TMS) & 1084.61 & 2.6124 & 0.027374 \\
\hline 21 & Glutamate & 1106.6 & 1.42183 & 0.035407 \\
\hline 22 & (2) & 1109.47 & 4.67479 & 0.028702 \\
\hline 23 & 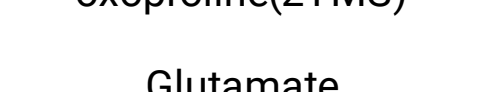 & 1129.86 & 1.57831 & 0.33655 \\
\hline 24 & GIt & 1197.65 & 1.07726 & 0.134376 \\
\hline 25 & Unknown Compounds ${ }^{a}$ & 1561.22 & 1.20351 & 0.008157 \\
\hline 26 & pinitol $^{b}$ & 1574.14 & 1.0647 & 0.110614 \\
\hline 27 & fructose & 1584.48 & 1.08737 & 0.111757 \\
\hline 28 & Methyl-glucopyranoside & 1586.28 & 1.00383 & 0.05818 \\
\hline
\end{tabular}




\begin{tabular}{|c|c|c|c|c|}
\hline 29 & Sorbose ${ }^{b}$ & 1592.42 & 1.10943 & 0.053829 \\
\hline 30 & Tyrosine(2TMS) & 1603.82 & 1.6122 & 0.938817 \\
\hline 31 & Glucose & 1648.58 & 1.37017 & 0.102376 \\
\hline 32 & Sorbitol & 1718.67 & 1.34052 & 0.405775 \\
\hline 33 & Inositol $^{b}$ & 1755.82 & 1.73995 & 0.390142 \\
\hline 34 & Inositol $^{b}$ & 1786.05 & 2.31075 & 0.088997 \\
\hline 35 & Palmitic acid & 1826.58 & 8.20016 & 0.60311 \\
\hline 36 & Inositol $^{b}$ & 1970.06 & 1.55279 & 0.130878 \\
\hline 38 & Oleic acid & $\begin{array}{l}1999.01 \\
2055.55\end{array}$ & $\begin{array}{c}1.37818 \\
1.5938\end{array}$ & $\begin{array}{l}0.123108 \\
0.191049\end{array}$ \\
\hline 39 & Stearate & 2067.1 & 1.74901 & 0.032301 \\
\hline 40 & Fructose-6-phosphate ${ }^{b}$ & 2314.72 & 2.69412 & 0.110923 \\
\hline 41 & Glucose-1-phosphate ${ }^{b}$ & 2452.07 & 1.02701 & 0.508436 \\
\hline 42 & $\begin{array}{l}\text { Inosine } \mathrm{e}^{\mathrm{b}} \\
\text { maltose }{ }^{\mathrm{b}} \\
\text { cholesterol }\end{array}$ & 2840.77 & 1.62726 & 0.819419 \\
\hline
\end{tabular}

Table 2.1. Contribution features was obtained from the PLS-DA Loading plot and variable importance in the projection (VIP) plot.RT;retention time.VIP; variable Importance in the Projection. P; results of t-test analysis.a; compounds that similarity is less than 800 , obtained from the comparative analysis of mass spectral library and spectra. b; compounds that similarity is greater than 800 , obtained from the comparative analysis of mass spectral library and spectra,but not comparison by the standard substance mass spectrum.Other compounds unlabeled: compounds that similarity is greater than 800 , obtained from the searching of mass spectral library and spectra, and comparison by the standard substance mass spectrum.

\section{Table2.2}




\begin{tabular}{|c|c|c|c|c|c|}
\hline number & Compounds & $\mathrm{RT}(\mathrm{s})$ & VIP & $\bar{P}$ & Model group \\
\hline 1 & lactic acid & 418.2 & 1.60741 & 0.005393 & $\uparrow$ \\
\hline 2 & alanyl-alanine $e^{b}$ & 864.525 & 2.71308 & 0.01851 & $\uparrow$ \\
\hline 3 & glutamic acid & 1106.6 & 1.42183 & 0.035407 & $\uparrow$ \\
\hline 4 & oxoproline & 1109.47 & 4.67479 & 0.016457 & $\uparrow$ \\
\hline 5 & pinitol $^{b}$ & 1561.22 & 1.20351 & 0.008157 & $\downarrow$ \\
\hline 6 & Glucose-1-phosphate ${ }^{b}$ & 2067.1 & 1.74901 & 0.032301 & $\downarrow$ \\
\hline
\end{tabular}

Table 2.2 Differences metabolites and change tendency of rabbit intervertebral disc degeneration (RT;retention time.VIP; variable Importance in the Projection. P; results of t-test analysis.a; compounds that similarity is less than 800 , obtained from the comparative analysis of mass spectral library and spectra. b; compounds that similarity is greater than 800, obtained from the comparative analysis of mass spectral library and spectra,but not comparison by the standard substance mass spectrum. Other compounds unlabeled: compounds that similarity is greater than 800 , obtained from the searching of mass spectral library and spectra, and comparison by the standard substance mass spectrum.)

\section{Figures}


Figure 1.1

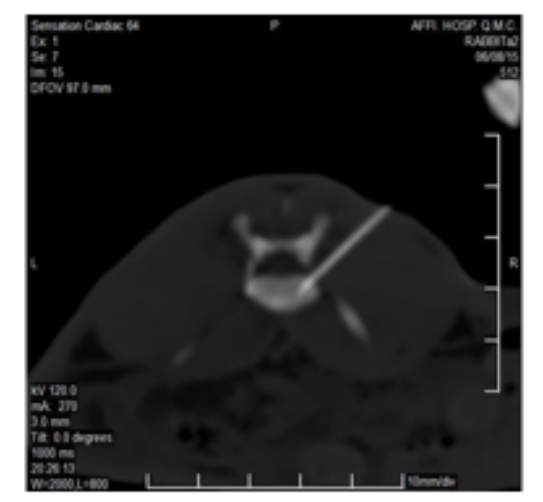

\section{Figure 1.2}

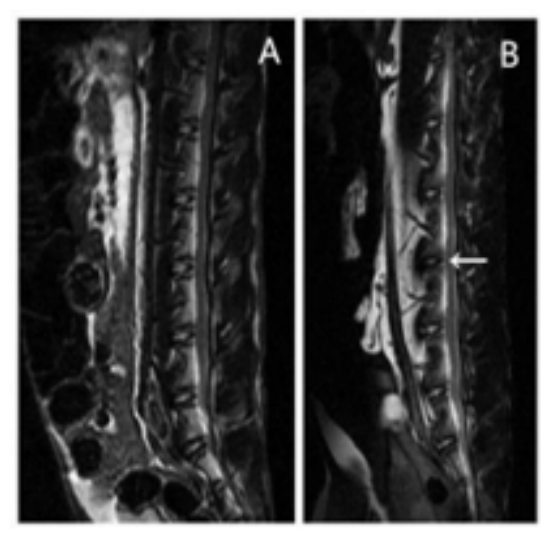

\section{Figure 1.3}

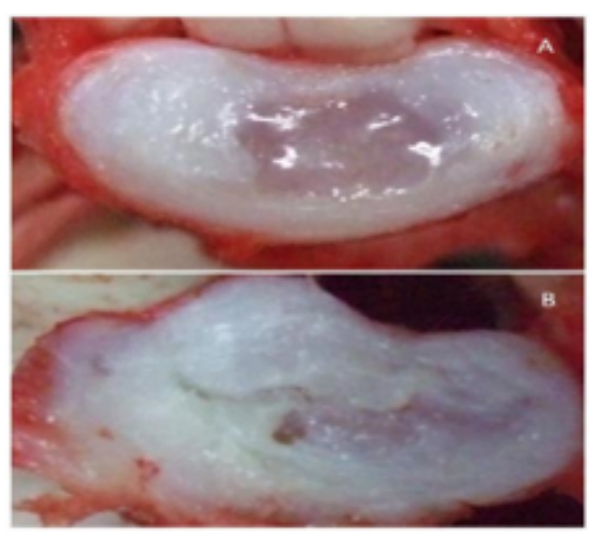

\section{Figure 1}

Figure 1.1,CT confirmed that the puncture needle had entered the nucleus pulposus. Figure 1.2, Control group (A) . Model group (B).Disc signal intensity was significantly decreased in model group at L4 / L5 (arrow L4 / L5 disc) Figure 1.3,A typical view of control group (A) and model group (B) 
Figure 2.1,
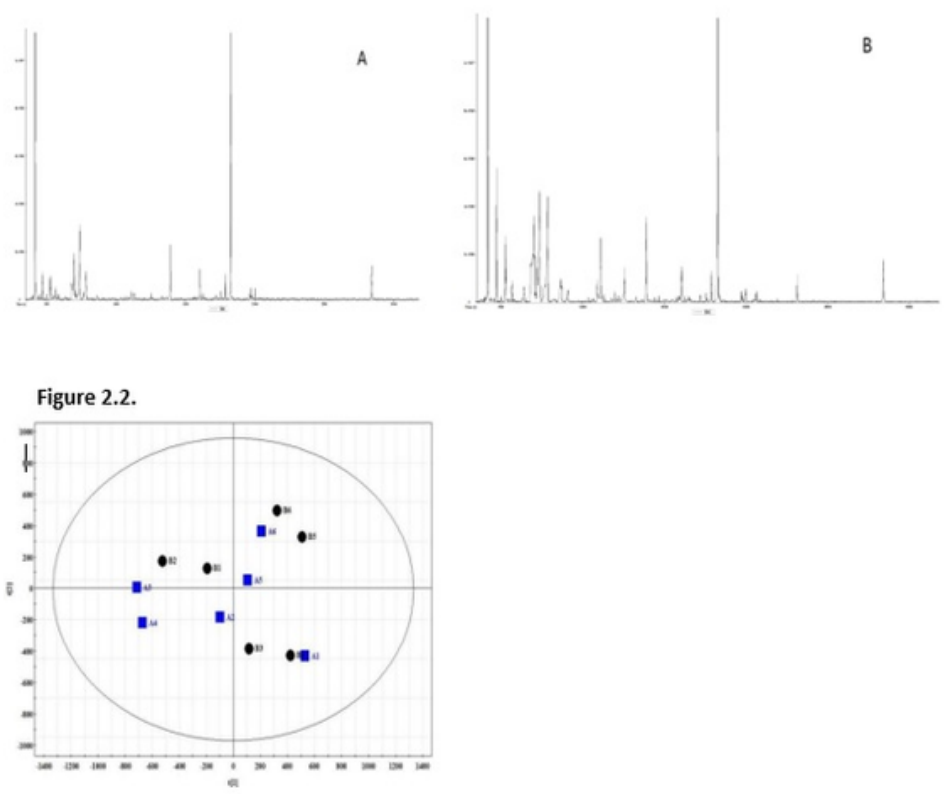

Figure 2.3.

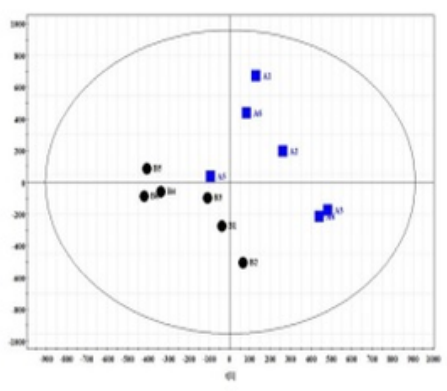

Figure 2.4

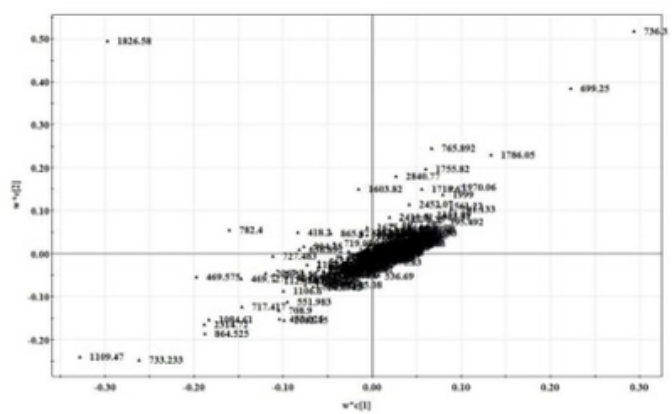

\section{Figure 2}

Figure 2.1,Two groups typical GC / TOF-MS TIC(A.Control group,B.Model group) Figure 2.2,PCA score plots of GC/TOF MS ( $\square$ :A.Control group,, :B.Model group ) Figure 2.3,PLS-DA score plots of GC/TOF MS (口:A.Control group, :B.Model group ) Figure 2.4,PLS-DA loading plot 
Fig3.

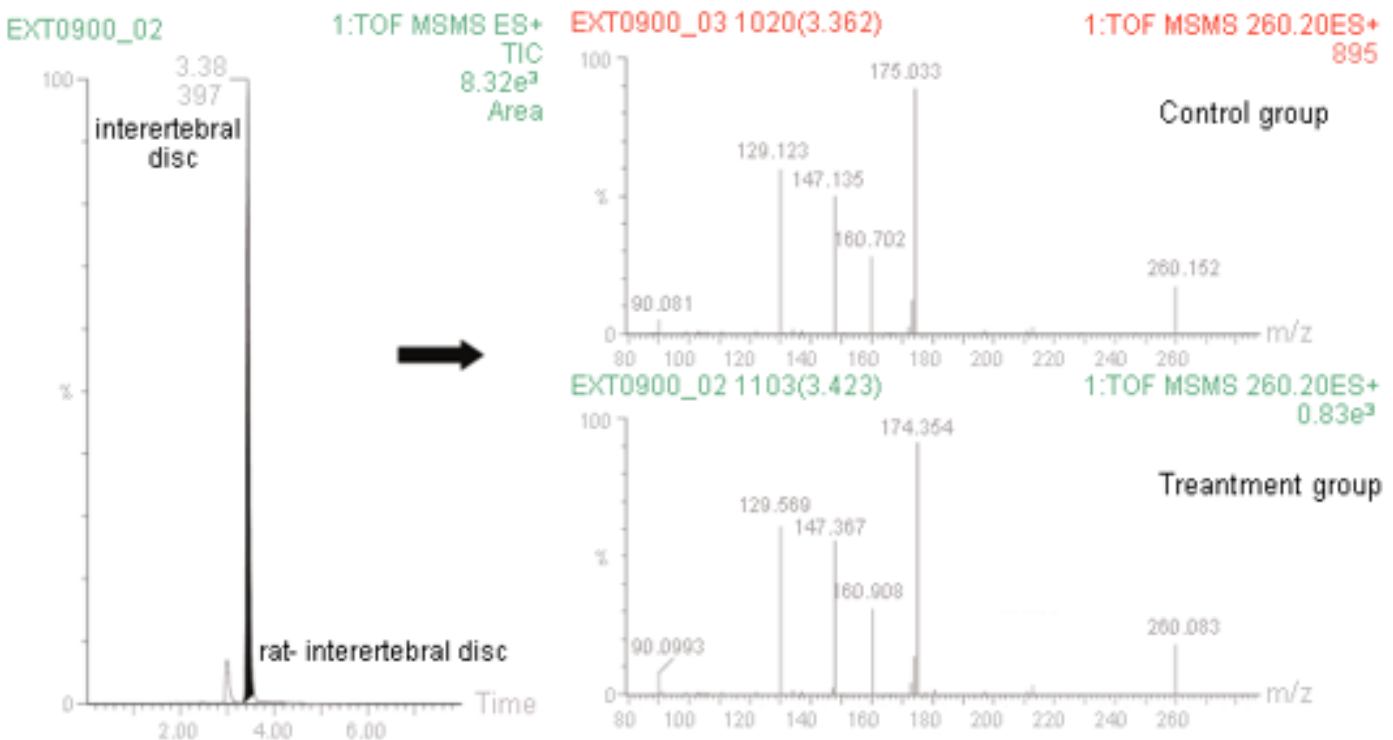

Figure 3

Significant differences in peak values of TiC spectra between two groups of disc samples as evaluated utilizing UHPLC-TOF and MS techniques

\section{Supplementary Files}

This is a list of supplementary files associated with this preprint. Click to download.

- NC3RsARRIVEGuidelinesChecklistfillable.pdf 